\title{
ECG Baseline Wander Reduction Using Linear Phase Filters
}

\author{
J. A. van Alste, W. van Eck*, and O. E. Herrmann \\ Twente University of Technology, Dept. E.E., P.O. Box 217, 7500 AE Enschede, The \\ Netherlands, and ${ }^{*}$ Dr. Neher Laboratories of the Netherlands Postal and Telecommunication \\ Service at Leidschendam, The Netherlands
}

Received November 19, 1985

The continuous real time reduction of baseline wander is a considerable problem in electrocardiography during exercises. Our solution consists of spectral filtering. The legitimacy of high-pass filtering of the ECG by means of digital linear phase filters with a low cutoff frequency as high as the heart rate is shown. The specifications of these filters are derived from experimental results. Special hardware is presented that simultaneously performs the desired real-time filter operation in four ECG leads. 1986 Academic Press, Inc.

\section{INTRODUCTION}

Baseline wander elimination is often one of the first steps required in the processing of the electrocardiogram (ECG). Not only before further automatic processing, but also as a first step in visual diagnosis. Reduction of baseline wander is especially required in exercise electrocardiography (XECG) and during ambulatory monitoring, because it is then that this wander is often pronounced.

Methods suggested for the removal of baseline wander in ECG's are (i) estimation and subsequent removal of the wander using polynomial splining techniques, and (ii) high-pass frequency spectrum filtering.

Zero- and first-order polynomials are commonly used for baseline wander estimation, both in visual diagnosis (for example, the use of a pencil and ruler in measuring ST-segment depression) and in automatic signal processing.

In the case of zero-order estimation, the average value of the PR segment of the ECG beat is used since this segment is by definition at baseline level. When the PR value exceeds a certain arbitrary threshold, the entire beat is often eliminated to inhibit measurements on distorted beats $(l)$. When estimating baseline wander by means of linear splining, the estimate is the straight line between two consecutive PR segments (2).

Meijer (3) describes a fast and elegant technique for the computation of a third-order polynomial through consecutive PR segments. This cubic spline technique gives a rather good estimation of the baseline signal in normal ECG 
registrations. The methods described above assume that the PR segments are well defined, recognizable, and that their position is known as is the case in normal beats. When not, the splining methods fail.

In order to be able to remove baseline wander continuously in real-time we investigated linear phase filter techniques. The wander can be removed by means of spectral separation provided that the frequency spectrum of the "true" ECG is not affected. A digital high-pass filter was used to study the separation of the baseline wander and the ECG in the frequency domain. Because this method is not discussed in the literature its possibilities were investigated. The results were used to design an applicable filter, implemented in specially designed hardware.

\section{Digital Linear Phase Filtering}

In the case of a nonrecursive digital filter with a finite impulse response (NRFIR) of length $N$ the convolution sum describing the filter operation can be written as

$$
y(n T)=\sum_{k=0}^{N-1} h(k T) \cdot x(n T-k T)
$$

where $y(n T)$ is the output signal

$x(n T)$ is the input signal

$h(k T)$ is the impulse response of the filter

$T$ is the constant sampling interval

$n$ is the actual sample index

$N$ is the number of coefficients of the impulse response.

If an impulse response with length $N$, where $N$ is considered odd, is chosen with an even symmetry around a certain point $M=(N-1) / 2$ thus $h(k T)=h([N$ $-k-1] T)$ then the corresponding spectral transfer function $H\left(e^{j \omega T}\right)$ of $h(k T)$ will have an exact linear phase (linear with respect to frequency (4)). This means that the various frequency components of the input signal are all delayed at precisely the same time. When this is not the case it is called phase distorsion which is a common property of analog filters.

\section{Spectral Separation of Baseline Wander from the ECG}

\section{Method}

Estimation of the power spectral density function of ECG registrations is a possible method for determining the spectral components of the ECG, including noise. However, the problem then arises to decide which components or parts of spectral components are from the "true" ECG and which are from the baseline wander. Therefore another method for evaluation of the influence of high-pass filtering on XECG was used. We applied a digital linear phase filter with a sharp edge between the pass and stop band to various exercise ECG registrations. Variation of the low cut-off frequency enabled us to distinguish how removal of certain spectral components effects the ECG. 
We made plots of 50 epochs each of $16 \mathrm{sec}$ of exercise ECGs obtained from different subjects. These epochs consist of the recorded ECG, the filtered ECG and a signal representing the difference between them. It could then be decided by visual inspection of those plots whether a component is present in the difference signal, which is correlated with the occurrence of beats. If so we can expect the "true" ECG to be mutilated. We chose this arbitrary criterium, since a suitable mathematical decision rule for these signals was not available.

The 50 epochs of ECG were chosen from recordings obtained during rowing ergometry (5). This type of ergometry is used in patients unable to perform leg exercise. The patient is seated in a chair and turns two parallel cranks against a torque that increases with the time. The exercise is similar to rowing a boat. Such ECGs often show a large amount of baseline wander, due to the trunk movement of the subject, as well as an alternating noise level due to myopotentials generated by the alternately active muscles in the thorax area. The observed registrations are obtained either from differential lead CC5 or from CM5 (6). Signal epochs, containing aberrancies, ST-segment depressions and much baseline wander were included in this study.

An analog fourth-order Butterworth low-pass filter with a $-3 \mathrm{~dB}$ cut-off frequency of $100 \mathrm{~Hz}$ was used to prevent aliasing of higher frequency components before digitizing the ECG at a sampling frequency of $250 \mathrm{~Hz}$ into 12-bit numbers.

We used digital linear-phase high-pass filters (Fourier approximation) (4) with a finite impulse response length of 1023 sampling intervals corresponding to $4.1 \mathrm{sec}$. The low frequency part of a typical transfer function is shown in Fig. 1. The filter shown has a low cut-off frequency of $1.14 \mathrm{~Hz}$ at $-0.5 \mathrm{~dB}$. The ripple in the passband of the filter (due to the Gibbs phenomenon) is less than $\pm 0.5 \mathrm{~dB}$, and the attenuation in the stopband is more than $40 \mathrm{~dB}$.

\section{Results}

Some results of the high-pass filtering are demonstrated in Figs. 2 to 7. Note that the exact time delay of $2.05 \mathrm{sec}$ between input and output due to the filter operation is eliminated for visual inspection purposes.

Figure 3 shows the influence of high-pass filtering on an ECG signal that shows a considerable ST-segment depression. The ECG epoch begins at a moment where the patient stops exercising resulting in a decreasing heart rate. In the difference signal the occurrence of components correlated with the ECG beats can be seen where the heart rate decreases to below the cut-off frequency of the applied filter $(1.85 \mathrm{~Hz}$ corresponding with $111 \mathrm{bpm}$.).

In some registrations the cut-off frequency could even be little higher than the heart rate without affecting the "true" ECG. Figure 4 shows an example where we also can see that the respiratory baseline wander is removed, but the respiratory influence on the R-wave amplitude remains unchanged.

The influence of high-pass filtering was also studied on ECG recordings containing (i) ST-segment depressions, (ii) ventricular extra systolae at random 


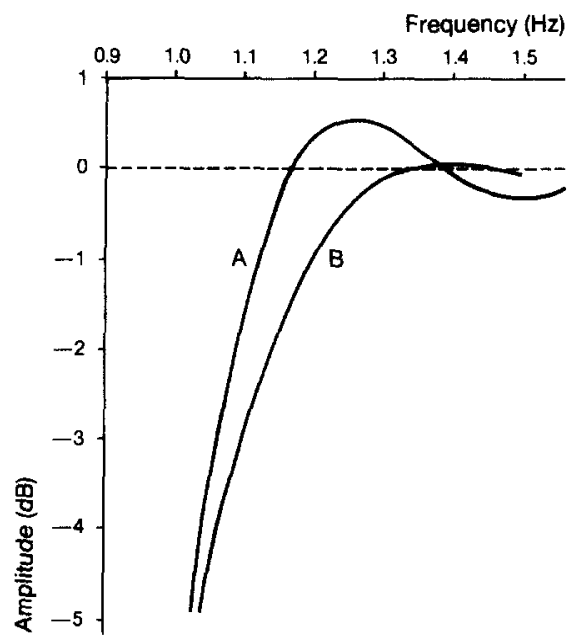

Fig. 1. Typical low frequency part of a transfer function of an allmost "ideal" high-pass filter for removal of baseline drift from the ECG. The impulse response length is $4.1 \mathrm{sec}$. Transfer function A is obtained by simple Fourier approximation and needs no improvement because the pass band ripple amplitude does not exceed $0.5 \mathrm{~dB}$. Transfer function $B$ shows the same filter after windowing with a Kaiser window. Here the pass band ripple amplitude is less than $0.1 \mathrm{~dB}$, but the transition edge between passband and stop band is less steep.

times (with and without compensatory pauses), (iii) multifocal ventricular doublets, (iv) bigemini, (v) bundle branch blocks, and (vi) nodal conduction disturbances (AV blocks of different degrees; sometimes with nodal extrasystolae).

The cut-off frequency of the high-pass filter was always chosen at the heart beat frequency or, when irregular of undefinable, the sinoatrial trigger repetition frequency (P-wave rate) or the waveform repetition frequency. A typical result is shown in Fig. 5.

We did not find any ECG recording in which a nonectopic beat was distorted due to the removal of spectral components below the actual heart frequency. We did, however, find minor changes in the shape of ventricular beats (Figs. 5 and 6). It was concluded from these results that a high-pass linear phase filter may have a cut-off frequency $(-0.5 \mathrm{~dB})$ up to just below the heart beat frequency, thus having no influence on the nonectopic ECG beats while removing baseline wander.

\section{Filter Specifications for Real-Time ECG Processing}

An advantage of high-pass filtering over other methods for baseline wander removal is the possibility of real time correction of the ECG. Then the filtered ECG is directly available after a short constant delay. We designed and realized equipment able to perform the filter operation in real time. In order to avoid considerable design complications a fixed low-cut off frequency of $0.8 \mathrm{~Hz}(-0.5$ dB) was chosen instead following the actual heart rate. This choice implies that the "true" ECG is not affected as long as the heart rate is higher than 48 bpm 


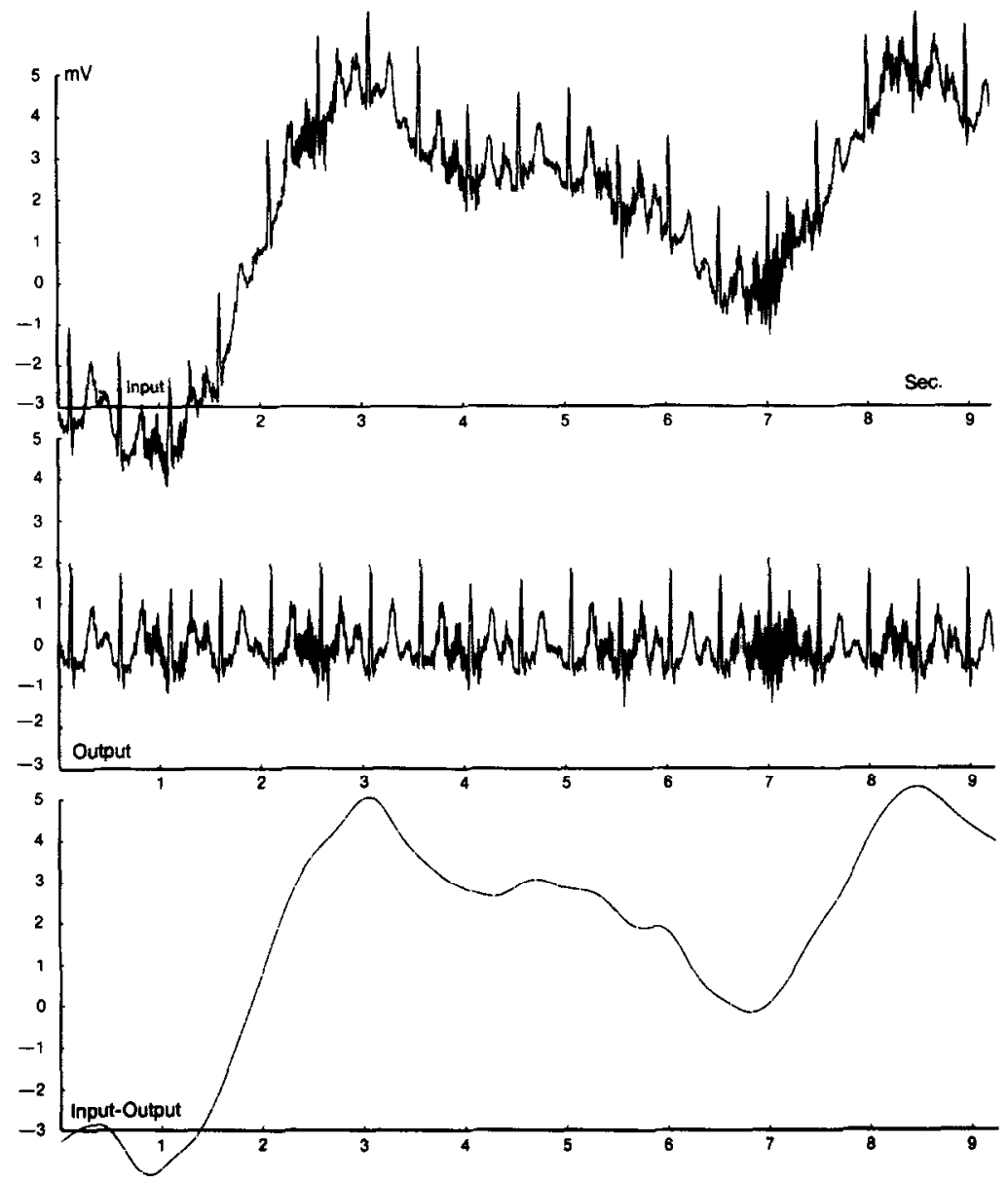

FIG. 2. The effect of high-pass NRFIR filtering on an ECG with much baseline wander and a high level of myopotential noise caused by arm exercise. The top tracing is the input ECG signal. The second tracing is the filter output (low cut-off frequency $1.9 \mathrm{~Hz},-0.5 \mathrm{~dB}$ ). The bottom tracing shows the part of the ECG of the top tracing which is removed by the filter. (Rectangular window; filter length 1023 sample points.)

(bradycardia). A high cut-off frequency of $100 \mathrm{~Hz}$ was used for removal of high frequency noise. Another important choice was that of a suitable impulse response to which the steepness of the edge between pass and stop band is proportional. A symmetrical impulse response filter causes a signal delay of half the duration of the impulse response. The computation time of the filter is proportional to the filter length. A length of $1.016 \mathrm{sec}$ was in every respect acceptable. It contains 255 coefficients given a sample frequency of $250 \mathrm{~Hz}$, and introduces an exact delay between input and output of $0.508 \mathrm{sec}$.

The coefficients of the impulse response were determined by computer-aided design techniques (7). We used a Kaiser weighted Fourier approximation (4) of a filter with a minimum stopband attenuation of $30 \mathrm{~dB}$. This attenuation reduces 

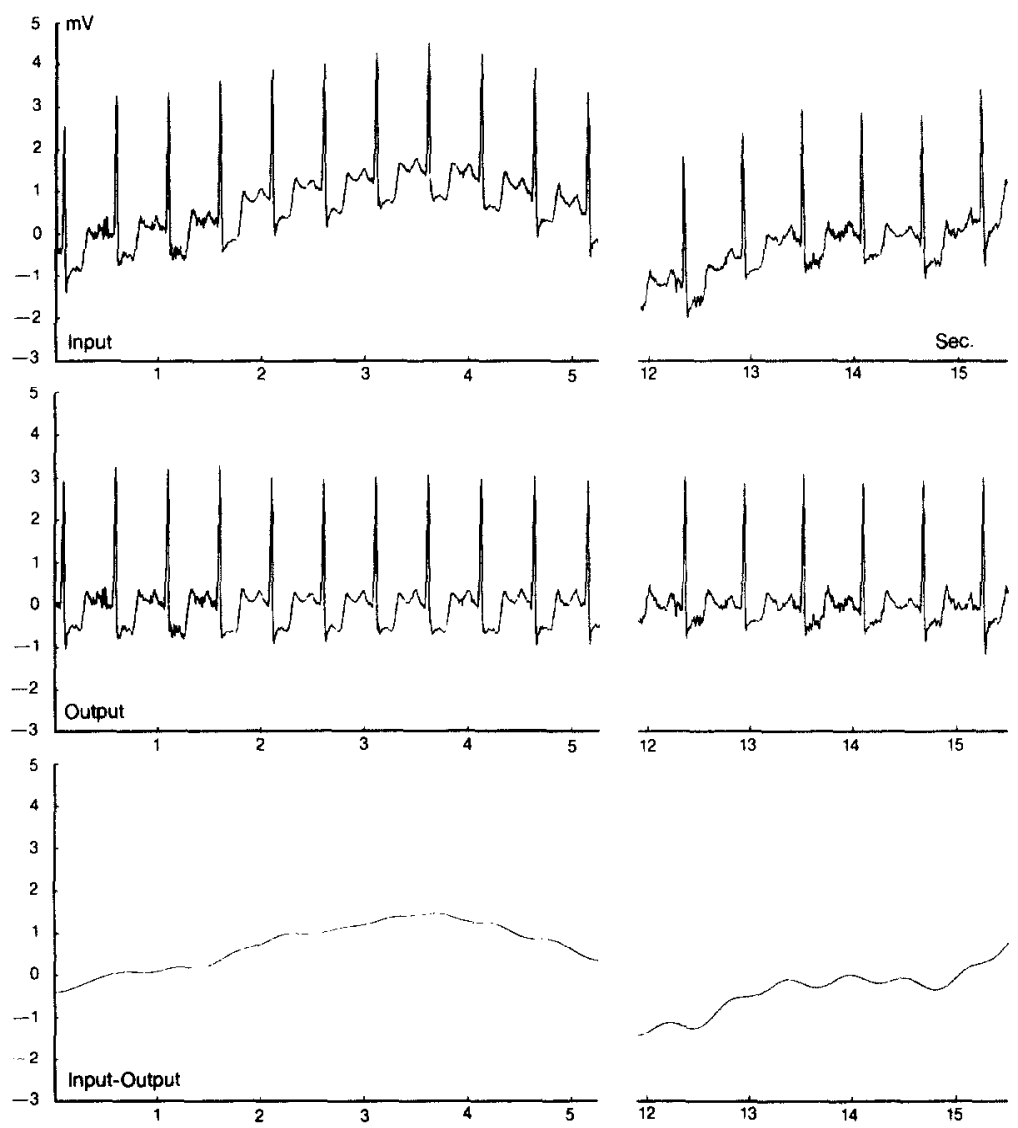

FIG. 3. The effect of high-pass filtering on an ECG with a considerable ST-segment depression. Note that the heart rate decreases with time. Components of the "true ECG" can be seen in the removed part of the signal (bottom tracing) when the heart rate is below $111 \mathrm{~min}^{-1}$ (corresponding to $1.85 \mathrm{~Hz}$ ). (Rectangular window; filter length 1023 sample points. Low cut-off frequency 1.85 Hz.)

a 3-mV baseline wander amplitude to less than $0.1 \mathrm{mV}$. The Kaiser windowing was necessary to reduce the passband ripple amplitude to less than $0.5 \mathrm{~dB}$.

The spectral transfer function is the obtained filter is shown in Fig. 7.

\section{A Hardware Filter for Real-Time Processing of the ECG}

It was necessary to process three ECG channels in parallel, in order to be able to use the filter for the orthogonal Frank lead system. The total number of 191, 250 multiplications needed, per second, cannot be calculated in real-time on a microcomputer. Therefore it was decided to design special equipment for this purpose.

Figure 8 shows a simplified block diagram of the realized filter hardware. This filter is able to process four ECG channels in parallel. The first processing step is analog low-pass filtering to prevent aliasing of high-frequency signal 


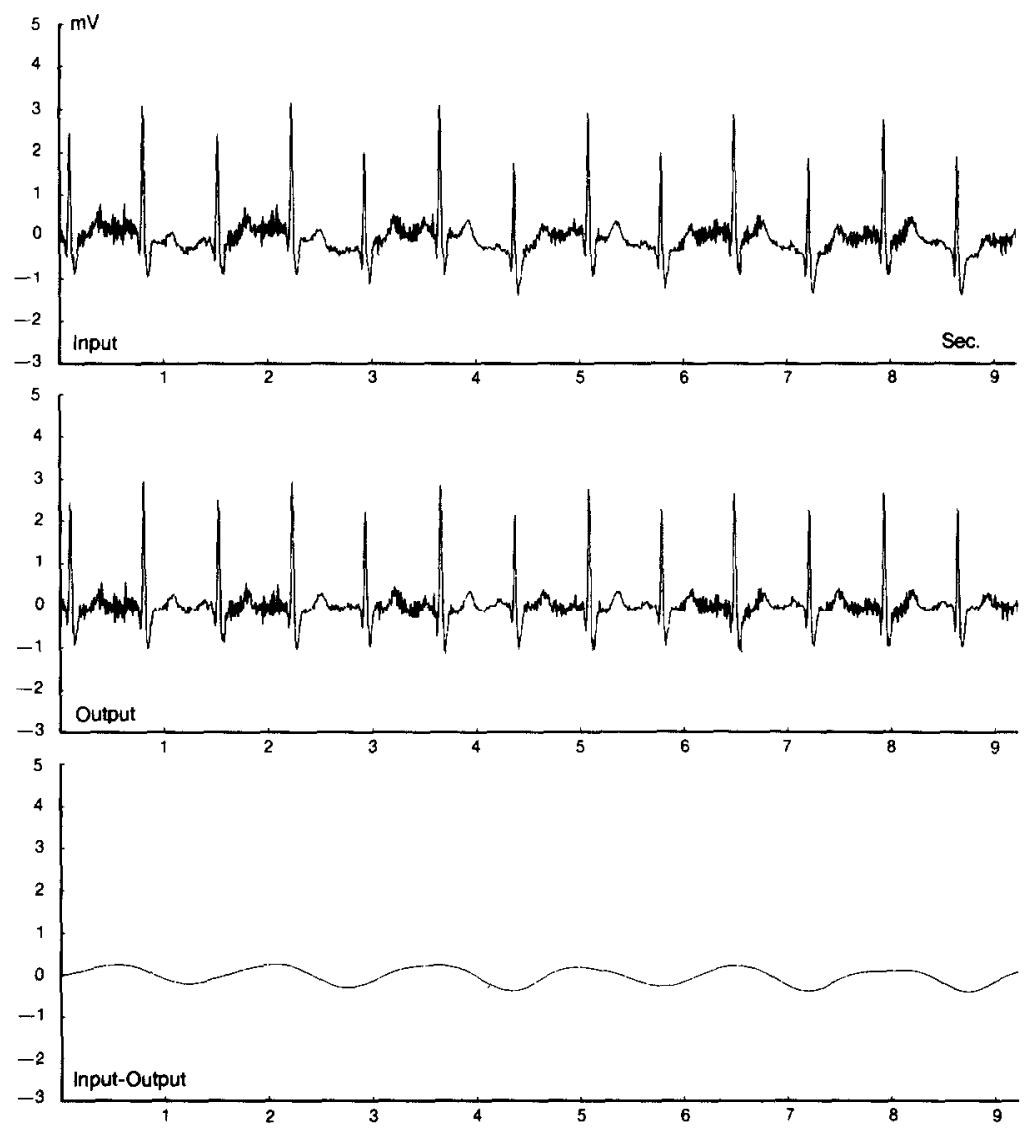

FIG. 4. The removal of respiratory bascline noise without affecting the respiratory influence on the R-wave amplitude. (Rectangular window; filter length 1023 sample points. Low cut-off frequency $1.50 \mathrm{~Hz}$.)

components after sampling. The four input channels are converted every 4 msec into 12-bit samples, which are stored in four cyclic RAM buffers (vector addressing). A buffer is read every millisecond and the convolution sum of the last 255 samples of the input signal and the corresponding filter coefficients is computed. Therefore the filtered sample values of all input signals are obtained in $4 \mathrm{msec}$.

The filter coefficients are stored in 16-bit precision in an EPROM that contains four different filter impulse responses of which one is selected by means of a front panel switch.

The convolution operation is carried out by a simple arithmetic unit. Its multiplying part consists of two $8 \times 8$ bit multiplier integrated circuits which presents the result of the $12 \times 16$ bit multiplication as sequential data. In this way the summation of all products is carried out by a 1-bit adder into a 30-bit shift register. The final result is truncated to 12-bit accuracy and outputted via 


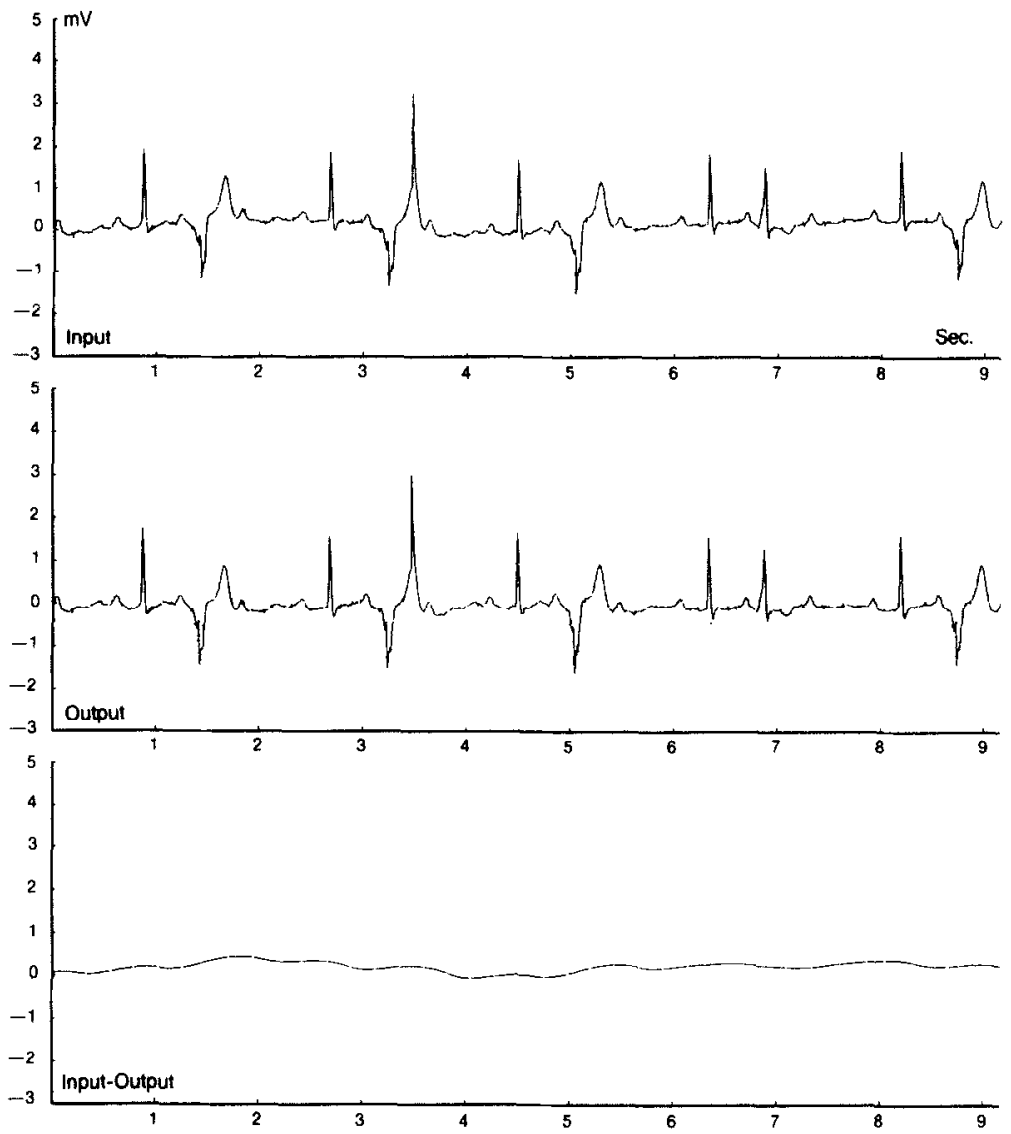

FIG. 5. An irregular ECG with ectopic aberrant beats that are hardly influenced by filtering. Only minor changes can be seen in the removed part of the signal (bottom tracing) when aberrant beats occur. (Rectangular window; filter length 255 sample points. Low cut-off frequency $1.10 \mathrm{~Hz}$.)

digital to analog converters and the signals are smoothed using analog reconstruction filters. For further digital processing the results are also made available to a digital output.

Figure 9 shows the influence of this filter on a typical exercise ECG recording as used in real time. Note that the output signal is delayed over $0.512 \mathrm{sec}$ compared to the input signal.

\section{Discussion}

An actual problem for discriminating between baseline wander and the "true" ECG is the lack of an objective decisive rule. Therefore we used visual inspection and searched for patterns in the parts of the ECG removed by the filtering that are correlated in time with the actual heart rate. We did not find such patterns when the low cut-off frequency of the filter was chosen below the actual heart rates, but we did find them when this was not the case.

When conforming to the American Heart Association recommendations for 

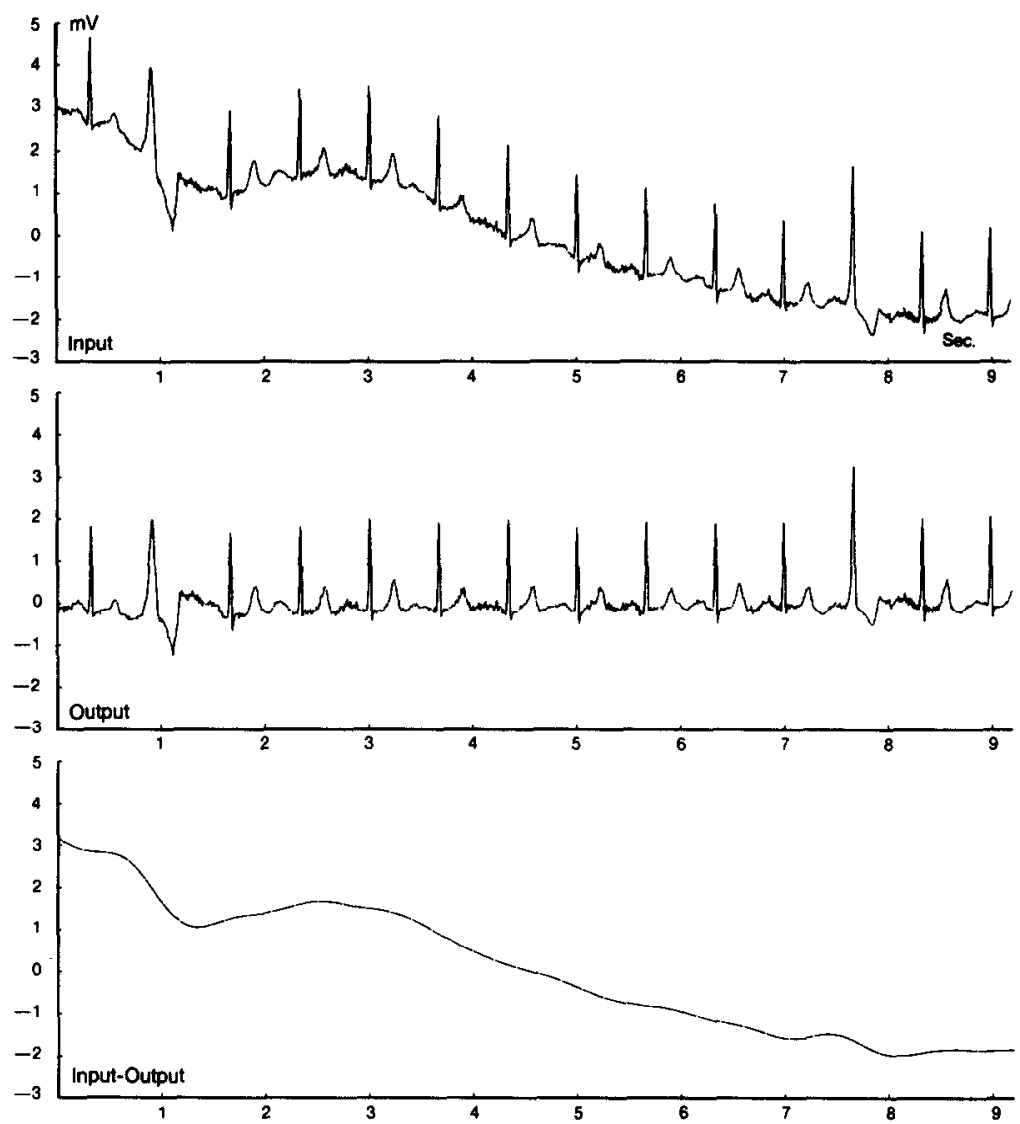

FIG. 6. An ECG recording where the aberrant beats are influenced by high-pass filtering. (Rectangular window; filter length 1023 sample points. Low cut-off frequency $1.40 \mathrm{~Hz}$.)

ECG recordings frequency components above $0.05 \mathrm{~Hz}$ should not be removed (8). Evidently these recommendations have been based on filter methods that introduce phase distortions above the heart-beat frequency. This is the case in analog filters and recursive digital filters with baseline suppressing properties. Such high-pass filters may have acceptable phase properties above the heart rate when their low cut-off frequency is chosen for example 10 times lower, at for instance $0.05 \mathrm{~Hz}$, as advised by the American Heart Association.

When comparing high-pass filtering with polynomial splining techniques, it is essential that spline techniques need reference points in the ECG signal. Generally the PR segment is used, and if this segment is not found the method fails. In the case of ectopic beats the PR segment is difficult to find or undefinable. This makes this method unsuitable for automatic real-time processing, where it is not known what the properties of the ECG will be.

High-pass filtering is very suitable in real-time processing, although the desired filter properties can only be obtained using rather extensive computational techniques. The number of multiplications can be reduced using specific 


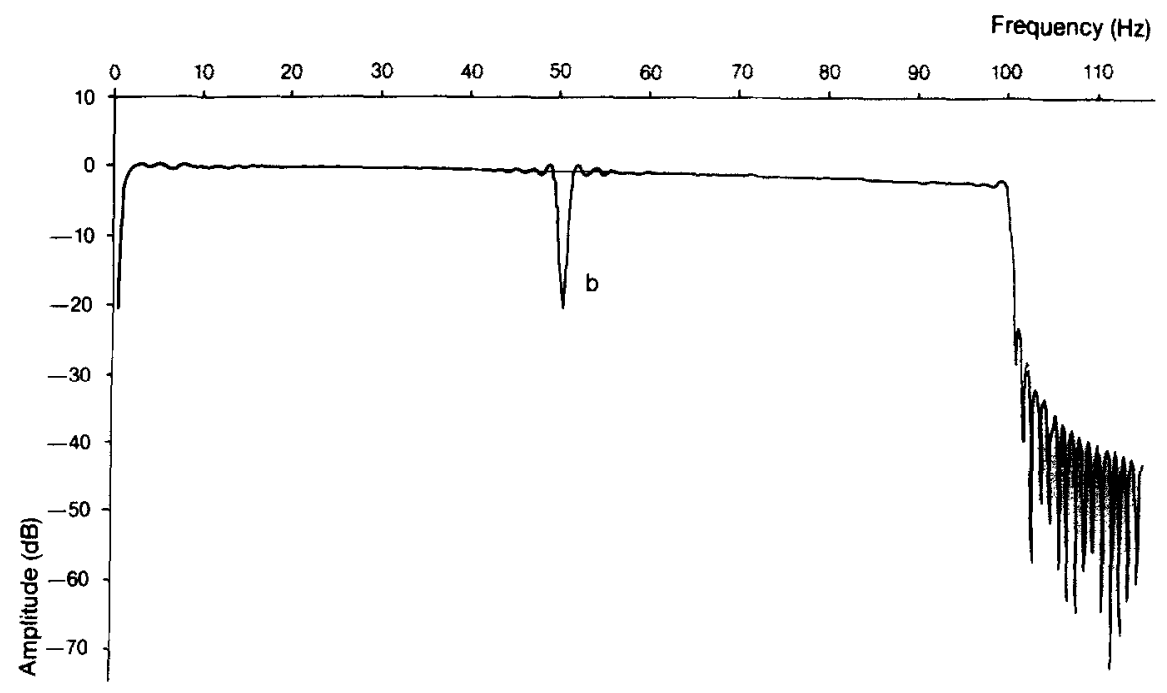

FIG. 7. A typical spectral transfer function of a NRFIR filter with a linear phase characteristic for the removal of baseline wander. The notch at $50 \mathrm{~Hz}$ (b) for removal of mains-frequency disturbances is optional.

properties of the applied impulse response (9). The signal samples that must be multiplied by equal filter coefficients can be summated before multiplication. In case of a symmetrical impulse response, each coefficient value appears at least twice.

\section{CONCLUSION}

It has been demonstrated that high-pass filtering is a helpful tool for removing baseline wander from exercise electrocardiograms, provided the filter has linear phase characteristics. The low cut-off frequency $(-0.5 \mathrm{~dB})$ of the filter can be as high as the heart-rate.

Baseline wander can be removed effectively in real time using a fixed low cut-off frequency of $0.8 \mathrm{~Hz}(-0.5 \mathrm{~dB})$. The filter operation can be performed by an apparatus such as the one presented in this paper.

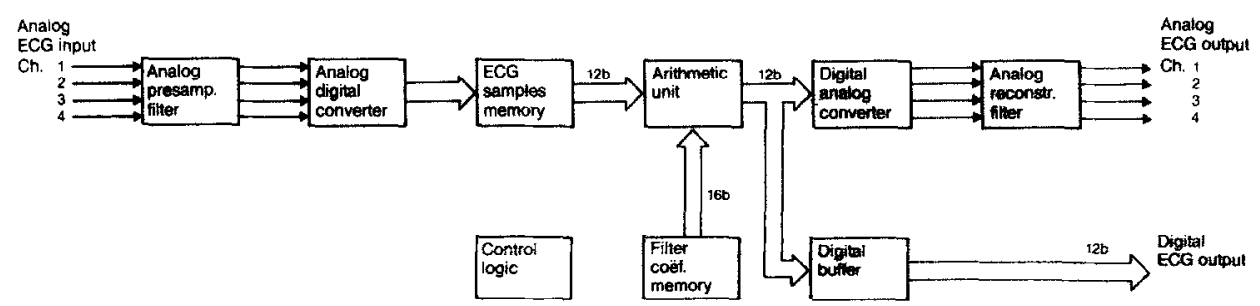

FIG. 8. Simplified block diagram of an apparatus for the nonrecursive finite-impulse response digital filtering of four ECG signals simultaneously in real-time. Refer to the text for details. 


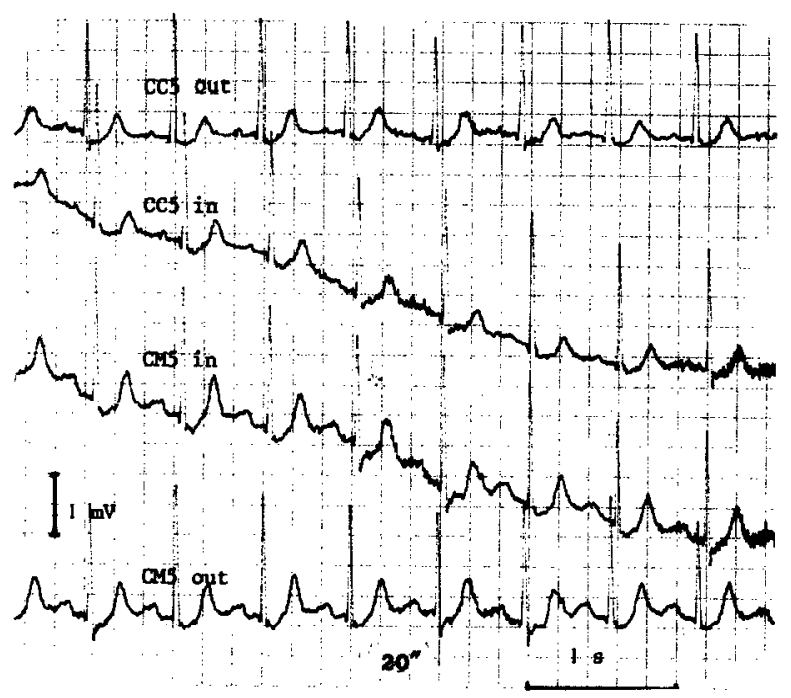

FIG. 9. The effect of the hardware implemented filter on an exercise ECG. The hardware is presented in Fig. 8, and its frequency response is presented in Fig. 7. Note that the output signals (top and bottom tracing) are delayed over $0.512 \mathrm{sec}$ compared to the input signals (middle tracings).

\section{REFERENCES}

I. Simoons, M. L., Boom, H. B. K., And Smallenburg, E. On-line processing of orthogonal exercise electrocardiograms. Comput. Biomed. Res. 8, 105-117 (1975).

2. Pipberger, H. Computer analysis of electrocardiograms. In "Computers in Biomedical Research" (B. Waxman and R. Stacy, Eds.), Vol. 1, p. 382. Academic Press, New York, 1965.

3. Meufer, C. R., AND KeISER, H. N. Electrocardiogram noise estimation and removal using cubic splines and state-space computation techniques. Comput. Biomed. Res. 10, 459-470 (1977).

4. Oppenheim, A. V., and Schafer, R. W. "Digital Signal Processing." Prentice-Hall, 1975.

5. van Alsté, J. A., Cruts, H. E. P., Huisman, K., and de Vries, J. Exercise testing of leg amputees and the result of prosthetic training. Int. Rehab. Med. 7, 93-98 (1985).

6. Ascoop, C. A., Distelbrink, C. A., AND De LANG, P. A. Clinical value of quantitative analysis of ST slope during exercises. Brit. Heart J. 39, 212-217 (1975).

7. Herrmann, O. E. Entwurf nicht rekursiver diskreter Systeme mit linearer Phase bei Vorschriften im Frequenzbereich. In "Digitale Systeme zur Signalverarbeitung” (H. W. Schüssler, Ed.). Springer-Verlag, 1975.

8. American Heart Association Committee on Electrocardiography. Recommendations for Standardization of leads and of specifications for instruments in ECG/VCG. Circulation 52, 11-25 (1975).

9. VAn Alsté, J. A., AND Schilder, R. S. Removal of baseline wander and powerline interference from the ECG by an efficient FIR filter with a reduced number of taps. IEEE Trans. Biomed. Eng. BME 32, 1052-1060 (1985). 